\title{
Neurorestorative Effects of Constraint-Induced Movement Therapy after Stroke: An Integrative Review
}

\author{
Marina Lucas $^{1}$, Pedro Ribeiro ${ }^{1,2,3}$, Mauricio Cagy ${ }^{4}$, Silmar Teixeira ${ }^{1,5,6}$, Fernanda Chaves ${ }^{1}$, \\ Diana Carvalho ${ }^{1}$, Caroline Peressutti ${ }^{1,3}$, Sérgio Machado ${ }^{7,8}$, Juliana Bittencourt ${ }^{1}$, Bruna Velasques ${ }^{1,3}$, \\ Roberto Piedade $^{1}$ \\ ${ }^{1}$ Brain Mapping and Sensory Motor Integration, Institute of Psychiatry of Federal University of Rio de Janeiro (IPUB/UFRJ), Rio de \\ Janeiro, Brazil; ${ }^{2}$ School of Physical Education, Bioscience Department, Federal University of Rio de Janeiro (EEFD/UFRJ), Rio de \\ Janeiro, Brazil; ${ }^{3}$ Institute of Applied Neuroscience (INA), Rio de Janeiro, Brazil; ${ }^{4}$ Division of Epidemiology and Biostatistic, Insti- \\ tute of Health Community, Federal Fluminense University (UFF), Rio de Janeiro, Brazil; ${ }^{5}$ Physiotherapy Laboratory, Veiga de \\ Almeida University (UVA), Rio de Janeiro, Brazil; ${ }^{6}$ Physiotherapy Department, Piquet Carneiro Policlinic, State University of Rio \\ de Janeiro (UERJ), Rio de Janeiro, Brazil; ${ }^{7}$ Laboratory of Panic and Respiration, Federal University of Rio de Janeiro (UFRJ), Rio de \\ Janeiro, Brazil; ${ }^{8}$ Physical Activity Neuroscience Laboratory, Salgado de Oliveira University, Rio de Janeiro, Brazil. \\ Email: silmar_teixeira@yahoo.com.br
}

Received July $15^{\text {th }}, 2013$; revised August $15^{\text {th }}, 2013$; accepted September $10^{\text {th }}, 2013$

Copyright (C) 2013 Marina Lucas et al. This is an open access article distributed under the Creative Commons Attribution License, which permits unrestricted use, distribution, and reproduction in any medium, provided the original work is properly cited.

\begin{abstract}
Stroke has been considered as one of the main causes of death and of motor and cognitive sequels. Especially, many patients with upper limb hemiparesis improved their motor action and showed meaningful cortical changes after treatment with constraint-induced movement therapy. Therefore, this review aims to verify the literature about neuroimaging and behavioral evidences in the cortical reorganization through the use of the constraint-induced movement therapy. So, we conducted the literature research in indexed journals from many databases like Pubmed, Medline, Cochrane Database, Lilacs and Scielo. We concluded that the behavioral and neuroimaging studies using traditional and modified constraint-induced movement therapy promote cortical reorganization.
\end{abstract}

Keywords: Stroke; Constraint-Induced-Movement-Therapy; Neuroplasticity; Cerebral Reorganization

\section{Introduction}

The cerebral vascular accident (CVA) is a frequent injury caused by the interruption of blood supply to the brain, resulting in oxygen deprivation. The CVA is one of the main causes of motor and cognitive impairments and death [1]. Particularly, upper limb hemiparesis, in many cases, has been the first complication of the vascular accident [2]. Experiments using cortical stimulation and functional image in animals and humans indicated that certain rehabilitation techniques tend to promote brain reorganization and improve upper limbs' activity [3]. Specifically, Constraint-Induced Movement Therapy (CIMT) $[4,5]$ has shown positive results in the treatment of upper limb motor sequels [5].

In the beginning, this treatment model was developed from observations in primates, which had their upper limbs deprived of motor action; later, this model was applied by Eduard Taub in hemiparesis patients during motor function recovery. This treatment was initially explored in order to observe changes in compromised adjacent cortical regions [6]. Since then, many studies have been conducted with the objective of improving the technical knowledge about the benefits of CIMT. It is known that this technique provides positive results regarding the improvement of the affected upper limbs, resulting in increased quality of life for the patient [5]. Researchers relate these behavioral gains with enlargement of the cortical excitability area $[7,8]$, and neurophysiologic principles and neuroimaging tools promote an understanding of how this reorganization of the central nervous system influences the recovery process of the individual [9].

The present literature review focuses on comparing new models of CIMT with the traditional view, in order to observe how much this new approach may benefit patients, and supposedly, the cortical changes were involved. Additionally, we present the foundations of brain research, and evidence linking cortical reorganization with 
CIMT. For this reason, we searched for English-written articles indexed in Pubmed, Medline, Cochrane, Lilacs and SciELO databases, using the key words "stroke", "stroke rehabilitation", "constraint-induced movement therapy”, "cortical reorganization” and "neuroplasticity”.

\subsection{Methodology}

We conducted a PubMed academic paper search focusing on articles written in English from 1985 to the present day (i.e., twenty eight years); only researches conducted with people and case-reports or original articles were included. Thus, for this integrative review, we employed the following search terms: constraint-induced movement therapy, modified constraint induced therapy, stroke, stroke rehabilitation, cortical reorganization, human brain, neurophysiology, neuroplasticity, hemiparesis and upper extremity. In our research, we combined the term "CIMT" with the afore-mentioned terms and we only selected the articles that reported the parietal areas as search term. Search results were then manually reviewed and the articles were considered for analysis; their relevance was determined by our consensus and by overall manuscript quality.

\subsection{Results}

We selected 23 articles with the combination of the terms "CIMT" and "CIMT modified" 14 articles with "stroke" and "stroke rehabilitation"; 13 articles with "cortical reorganization" and "human brain"; 13 articles with "neurophysiology” and "neuroplasticity”, 12 articles with "hemiparesis", and "upper extremity". After this selection, we used 75 articles which fulfilled the objective of the study.

\section{Types of Constraint-Induced Movement Therapy}

Experiments using behavioral assessments, cortical stimulation and neuroimaging tools showed us that these rehabilitation techniques generate positive results in the recovery of Stroke patients. [10,11]. A recent form of rehabilitation for these patients is constraint-induced movement therapy $[4,5]$. Specifically, the treatment developed by Edward Taub in the late 1970s and early 1980s consists in restricting the movement of the unaffected upper limb during $90 \%$ of the patient's awake time for a period of 14 consecutive days, and it involves specific training for about 6 hours a day for 10 consecutive days [12,13]. During the intervention, the patient wears a glove on the free hand that can be removed to perform activities such as washing hands and using the restroom [14]. Specific training, known as "shaping", is a conditioning method in which the difficulty of the motor tasks increases pro- gressively [15].

However, for many post-stroke patients, the traditional protocol can be hardly accessible, due to the intense workout routines, the program duration [16] and the cost of treatment in a specialized clinic. Thus, with the objective of facilitating the application of the technique, Page et al. [16] created a new protocol in which they decreased the intensive training session of the affected upper limb and the retention time of the unaffected upper limb. The training began to be conducted three days a week and at the same time they restricted the unaffected limb for 5 hours per day, 5 days a week for a period of 10 weeks, and they found positive results.

Therefore, a more accessible modified version of the CIMT protocol (mCIMT) has been proposed by some researchers $[16,17]$. The mCIMT also aims to the immobilization of the unaffected limb, conditioning the affected one; however, the periods of immobilization and attendance at the clinic are shorter [18]. The convenience of this procedure provided greater clinical implementation of the technique in a larger number of patients [16]. Furthermore, the effectiveness of the technique originally developed by Eduard Taub was not compromised after its modification. This has been observed in several studies showing that mCIMT can improve the ability of the paretic upper extremity [1,3] (Table 1).

\subsection{Neuroimaging and Electrophysiology Foundations}

More non-invasive neuroimaging techniques have been of crescent interest to examine the behavioral changes and neural mechanisms, thus resulting in the development of new approaches to improve motor rehabilitation [19]. The neuroimaging techniques have been considered as promising to assess cortical connectivity. Because of their importance, many studies have utilized these tools to improve knowledge about CIMT, understand the neurophysiological variables related to the application of the technique and ultimately provide a better understanding as to its benefits. Non-invasive brain research tools such as functional magnetic resonance imaging (fMRI), positron emission tomography (PET), electroencephalography (EEG) and transcranial magnetic stimulation (TMS) have been used to examine changes in the neural mechanisms during treatments with CIMT [19]. Therefore, the following paragraphs will provide a brief review of electro-physiological fundaments and their importance, in order to answer questions about the cortical changes caused by CIMT.

\subsubsection{Electroencephalography}

The electroencephalogram (EEG) records electrical currents from the variations of neuron's action potentials in the cerebral cortex. For this purpose, electrodes are placed 
Table 1. Studies developed using EEG, TMS, PET, fMRI tools and MAL, WMFT and FM scales.

\begin{tabular}{|c|c|c|c|c|}
\hline Study & $\mathbf{N}$ & Tool & CIMT protocol & Results \\
\hline Milter et al., [6] & 15 & MAL and WMFT & $\begin{array}{l}\text { 90\%REST- } \\
\text { 07hrsT.14D }\end{array}$ & Significant increase of upper limb functionality and affected hemisphere. \\
\hline Lipert et al., [7] & 13 & TMS & $\begin{array}{l}\text { 90\%REST } \\
\text { 06hrsT.12D }\end{array}$ & Significant increase of hand functionality and affected hemisphere. \\
\hline Sterr et al., [4] & 15 & MAL and WMFT & $\begin{array}{l}\text { 90\%REST- } \\
\text { 06hrsT. 14D. }\end{array}$ & $\begin{array}{l}\text { Significant improvements in motor function and increased use of the } \\
\text { affected hand. }\end{array}$ \\
\hline Schaechter et al., [61] & 4 & $\begin{array}{l}\text { MAL, FM and } \\
\text { fMRI }\end{array}$ & $\begin{array}{l}\text { 90\%REST- } \\
\text { 4hrsT.14D }\end{array}$ & Significant increase in cortical activation in affected hemisphere. \\
\hline Wittenberg et al., [59] & 16 & $\begin{array}{l}\text { MAL, WMFT, } \\
\text { PET and TMS }\end{array}$ & $\begin{array}{l}\text { 90\%REST- } \\
\text { 06hrsT. 10D. }\end{array}$ & $\begin{array}{l}\text { Cerebral activation during a motor task decreased significantly, and motor } \\
\text { map size increased in the affected hemisphere motor cortex. }\end{array}$ \\
\hline Könönen et al., [72] & 12 & PET & $\begin{array}{l}\text { 90\%REST- } \\
\text { 06hrsT. 14D. }\end{array}$ & $\begin{array}{l}\text { Significant increase of blood perfusion in pre-motor affected hemisphere; } \\
\text { cingulate unaffected hemisphere and in two cerebellum hemispheres. }\end{array}$ \\
\hline Szaflarski et al., [17] & 7 & $\begin{array}{l}\text { MAL, FMA and } \\
\quad \text { fMRI }\end{array}$ & $\begin{array}{l}\text { 5hrs REST- } \\
\text { 30minT. 10D }\end{array}$ & $\begin{array}{c}\text { Significant increase in cortical activation in affected and } \\
\text { unaffected hemisphere. }\end{array}$ \\
\hline Taub et al., [50] & 21 & MAL, WMFT & $\begin{array}{l}\text { 90\%REST- } \\
\text { 03hrsT. 14D. }\end{array}$ & Significant increase of affected upper limb functionality. \\
\hline Junger et al., [71]. & 10 & fMRI & $\begin{array}{l}\text { 90\%REST- } \\
\text { 06hrsT.12 D. }\end{array}$ & $\begin{array}{l}\text { Significant increase of affected hand functionality and more activation in } \\
\text { primary sensorimotor cortex in affected hemisphere. }\end{array}$ \\
\hline Page et al., [53] & 4 & MAL and FM & $\begin{array}{l}\text { 5hrs REST- } \\
\text { 1/2-hrT. 10D. }\end{array}$ & $\begin{array}{l}\text { Significantly increased use of the affected arm and } \\
\text { increased ability to perform valued activities }\end{array}$ \\
\hline Boake et al., [75] & 23 & $\begin{array}{l}\text { MAL, TMS and } \\
\text { FM }\end{array}$ & $\begin{array}{l}\text { 90\%REST- } \\
\text { 03hrsT. 14D. }\end{array}$ & $\begin{array}{l}\text { Improvement in affected hand motor function on the FM associated with a } \\
\text { greater number of sites on the affected cerebral hemisphere where responses } \\
\text { of the affected hand were evoked by TMS }\end{array}$ \\
\hline Wu et al., [56] & 6 & MAL and fMRI & $\begin{array}{l}\text { 2hrs/dia REST. } \\
\text { 2hrsT. } 3 \text { week }\end{array}$ & $\begin{array}{l}\text { Significant improvement in motor function of affected upper limb and } \\
\text { affected hemisphere with increase of the cortical activation. }\end{array}$ \\
\hline Tarkka et al. [25] & 13 & EEG & $\begin{array}{l}\text { 90\%REST- } \\
\text { 06hrsT. 14D }\end{array}$ & $\begin{array}{l}\text { Significant increase of affected hand functionality and more activation in } \\
\text { motor cortex, pre-motor cortex and the primary motor area in } \\
\text { both hemispheres. }\end{array}$ \\
\hline
\end{tabular}

on the scalp [20] at specific positions where changes of neural patterns are identified [21]. The neurons generate small electrical currents around cell membranes, specifically along the dendrites, and they receive input signals from other neurons [22]. These electrical activities result from the electrochemical communication among neurons and correspond to the information flow occurring in several cortical regions [21].

The EEG allows monitoring, identifying and classifying electrophysiological bioelectrical signals in frequency ranges, bands of activity, or brain rhythms, relating them to waking and non-waking states [23]. The power spectrum of clinical interest is generally considered starting from about $1-20 \mathrm{~Hz}$. This frequency range has been traditionally divided into frequency bands typically defined as delta (1.5 to $3.5 \mathrm{~Hz}$ ), theta (3.5 to 7.5 ), alpha (7.5 to $12.5 \mathrm{~Hz}$ ) and beta (12.5 to $20 \mathrm{~Hz})$. The recorded activity at each electrode can be represented as the absolute power in each band, relative power (percentage of total energy in each band), coherence (a measure of synchronization between the activity in two channels), or asymmetry (the power ratio in each band between a pair of symmetric electrodes) [24].
Due to the efficiency of this tool, many studies have been performed with the objective of investigating the cortical changes resulting from CIMT [25]. The answers to these questions are crucial to understand the mechanisms of the brain plasticity that may ultimately result in behavioral gains for the individual affected by stroke [15].

\subsubsection{Transcranial Magnetic Stimulation}

In 1985, Barker and colleagues [26] showed that it was possible to stimulate brain regions with little or no pain using transcranial magnetic stimulation (TMS). TMS is a non-invasive technique originally introduced to investigate neural propagation [27]. It is a procedure in which a magnetic field stimulates electrical activity in the brain [28]. It induces electrical currents in cortical neurons by placing a small coil on the scalp. Constant changing of these currents inside the coil results in the formation of a magnetic field, which is able to overcome different levels of various structures such as skin, muscle and bone [29].

The maximum force field generated by the stimulator is able to activate cortical neurons at a depth of 1.5 to 2 $\mathrm{cm}$ under the scalp [30]. It is assumed that magnetic 
stimulation (100 ms duration) excites a neural population, inducing rapid changes in the rate of neural networks for only a few milliseconds [31]. The magnitude of the induced current is dependent on both the magnitude and variation rate discharged through the coil; this current then produces a brief, but powerful electric field through the brain tissue, depolarizing neurons [30]. TMS has different intensities, frequencies and locations, so the stimulus probably occurs in different groups of neurons [32].

As a research tool, TMS has been applied in several studies [33,34]. For instance, Pallanti and Bernardi [35] reported that the use of TMS in the dorsolateral prefrontal cortex region reduces the symptoms of anxiety and panic disorder. Thus, it is necessary to clearly determine the role of TMS for rehabilitation and several studies have applied it to investigate cortical changes in subjects undergoing CIMT after stroke [36].

\subsubsection{Functional Magnetic Resonance Imaging (fMRI)}

Magnetic resonance imaging (MRI) is a technique that allows the visualization of three-dimensional high resolution tomographic images without ionizing radiation. This technology has also allowed the evaluation of pathological features in a non-invasive way [37]. Furthermore, MRI is widely used to study the functional organization (fMRI) of the human brain, in which neuronal signals recordings are presented in response to magnetic stimuli [38]. The fMRI is not geared specifically to investigate clinical aspects. It is also used to study healthy individuals and their brain functional changes [39].

The fMRI analysis is based on a technology that uses a strong magnetic field and radio waves to capture the hemodynamic and metabolic changes induced by neural activity, i.e. by local increases in the blood volume, flow and oxygenation [40]. This increase activates an intense magnetic field resulting from the alignment of its nuclear spins (orientation of subatomic particles when immersed in a magnetic field). So, when the radiofrequency pulse is launched on the representation of blood, the spins move from their original orientation to an excited energy state. During this process, the nuclear spins tend to return to their initial condition, i.e. the state of lowest energy; however, when this occurs, the excess energy is emitted in the form of electromagnetic radiation. This energy is detected by the fMRI device and allows the formation of anatomical images [39].

fMRI has been a widely used tool in brain mapping research. It allows the studying of neural networks dynamically by tracking specific responses in several spatial scales; however, fMRI can measure only hemodynamic changes such as variations in blood volume, or intravascular magnetic susceptibility [41]. Moreover, regardless of whether it is an incipient technique, fMRI has been applied to a variety of functional studies, ranging from simple to complex experiments such as neuropsychological investigations [39]. Thus, it stands out by allowing the exploration of various brain functions, due to its high ability to differentiate tissues. In particular, some researchers have used fMRI to observe cortical changes in subjects undergoing CIMT. Therefore, Kim et al. [42] observed changes in the neural plasticity of the premotor cortex and supplementary motor area. These studies are very important to understand the efficiency of the technique and its effects in post-stroke patients. This way, we can offer to patients a therapy to improve their functional limitations.

\subsubsection{Positron Emission Tomography (PET)}

Brain mapping techniques are vital for understanding molecular, cellular and functional mechanisms [43]. Among them, PET is an extremely effective tool for mapping functional organization in the human brain, contributing not only to rehabilitation, but also to research [44]. This technique produces a three dimensional image of functional processes in the organism [45]. In order to perform a scan, a short radioactive tracer isotope is injected in the subject (usually in the bloodstream). The tracer is chemically incorporated to a biologically active molecule, typically a substance such as glucose, which can be metabolized by the body cells. [46]. PET produces mainly functional and physiological information; anatomical structures are, in turn, difficult to identify. Therefore, PET usually has to be combined with anatomical methods such as computed tomography or magnetic resonance imaging [47].

\subsubsection{Advantages and Disadvantages}

There are several additional methods that can be used to study the contribution of specific cortical networks for cognitive and motor functions. For example, PET and fMRI are able to reveal brain networks involved in specific functions; however, these techniques have poor temporal resolution and cannot demonstrate per se whether a particular cortical area is essential for a specific function [47]. TMS, on the other hand, can inhibit, activate, and temporarily disrupt brain activity, allowing the function evaluation on a scale of milliseconds [30, $36,48]$. Yet, EEG studies typically suffer from poor spatial resolution with a relatively imprecise location of electromagnetic patterns associated with the neural current flow [38]. Notwithstanding, the low cost of the examination and its great ability to measure spontaneous brain activity seem to attract professionals to use this tool [21]. The high density of the EEG, combined with TMS, provides a direct and non-invasive measure of cortical 
excitability and connectivity in humans. These techniques may be used to track changes over time, such as pathological, plastic and therapy-induced modifications in cortical circuits [49].

\subsection{Effects of Constraint-Induced Movement Therapy on Post-Stroke Patients: Behavioral Results}

Several investigations have been conducted in order to analyze whether CIMT is effective in individuals with a hemiparetic upper limb after stroke [50]. Milter et al. [6] restricted the movement of the unaffected upper extremeity of 15 hemiplegics for $90 \%$ of the time when they were awake. Patients underwent the CIMT for 14 consecutive days with a daily 7 hour intervention. In this study they used Motor Activity Log (MAL) and Wolf Motors Functional Test (WMFT) scales to verify the functional condition of individuals. They executed the evaluations with this scale on five occasions: 1) 15 days before treatment, 2) one day before and 3) one day after treatment, 4) four weeks and 5) six months after treatment. In this experiment they concluded that the CIMT shows positive results in the hemiparetic upper limb rehabilitation in Stroke patients.

Kuntel et al. [51] conducted a study with five chronic and moderate motor deficit Stroke patients. They restricted the unaffected upper end of the limb with a sling for 14 days. The patients used the sling 6 hours per day and executed the task with the affected extremity. The researchers observed changes in the affected upper limb through the Motor Activity Log (MAL), Wolf Motor Function Test (WMFT) and Arm Motor Ability Test (AMAT) before and after treatment. They found large improvement in the amplitude of the movements, with significant changes in the scale results used to monitor the groups studied.

Edward Taub, one of the creators of CIMT, said that techniques that induce the patient to use the affected limb should be considered efficient because they stimulate cortical reorganization and increase the use of such limb [52]. Based on this information, several authors modified the applicability of the technique in order to facilitate its use for both the therapist and the patient [10,13,18,53, 54].

Thus, the mCIMT has various protocols; one of the most used is for 30 minutes a day, 3 days a week for 10 consecutive weeks with specific training for daily living activities. At home, the patient uses the immobilizer on the affected limb 5 hours a day, 5 days a week for 10 weeks $[17,18,54]$. In another protocol the patient trains 5 hours per day (2 morning hours and 3 afternoon hours) for two consecutive weeks. During these 5 hours, the patient performs physiotherapy tasks for 2 hours, and during the other 3 hours, he/she is instructed to perform a self-training at home [55]. Furthermore, the mCIMT has been used for 2 hours per day, five days per week for 3 weeks [56]. The mCIMT protocols described here have reported a significant improvement in the affected upper limb motor function $[1,8,56]$. Another experiment utilized the mCIMT for two weeks in 15 patients who were divided into two training groups. In such groups, the individuals remained with their limb restricted for $90 \%$ of the time during which they were awake. However, the first group trained 6 hours per day, while the second group trained three hours per day. They used two scales before and after intervention, the MAL and WMFT. Their results showed that in all scales mCIMT is an effective treatment in hemiparetic individuals; however, the benefits were greater in the group that trained 6 hours a day [4].

In the past, the effects of CIMT were studied in subjects who had been injured for more than one year, though the present studies suggest that also subacute patients who have suffered a stroke can benefit from the therapy [6]. Wolf et al. [57] after comparing the efficacy of treatment among subacute (3 to 9 months) and chronic (over 12 months) patients via behavioral scales such as Wolf Motor Function Test, Motor Activity Log and SIS, concluded that functionality improved in both groups; however, the group in which the technique is applied earlier shows significantly greater changes compared with the group in which the technique is applied later. This finding agrees with the study by Biernaskie et al. [58] which demonstrates that early rehabilitation provides functional recovery and greater plasticity compared with late rehabilitation.

Furthermore, several studies show that the affected limb remains in good conditions even after a long time [59,60,61]. Blanton and Wolf [62] studied the efficacy of CIMT after 3 months of therapy application and observed a continuous improvement in the patient. This result provides evidence that the increased functionality does not only occur immediately after treatment but it also appears to last for a long time. This study supports Milter et al. [6] who concluded through a behavioral analysis performed in three steps (immediately after the therapy application, 4 weeks and 6 months after treatment) that, even after some time, functionality does not decrease, and this also supports the hypothesis that CIMT produces a permanent improvement of motor functions.

In addition, Winstein et al. [60] evaluated the immediate and long-term effects using two rehabilitation approaches: strength and endurance training with the affected limb and a training of functional daily life activeties, but without hand immobilization. The study concluded that the immediate benefit of a functional approach was similar to the application of force and endur- 
ance, however, the former was more beneficial in the long term. This result underscores the importance of inserting the application of daily activities in CIMT, because this reduces the dependence of individuals [63].

\subsection{Effects of Constraint-Induced Movement Therapy on Post-Stroke Patients: Evidence through Cerebral Reorganization}

Several studies with CIMT and mCIMT have used electrophysiological analysis tools to observe the cortical changes which occur when this technique is employed [64]. In particular, most studies about Stroke using fMRI associated the gains in motor functions of the affected hand after CIMT with cortical activation increase in the ipsilateral hemisphere related to the hemiparesis $[56,61]$. The PET and fMRI have been useful tools in analyzing changes in post-Stroke patients who were treated with CIMT and mCIMT. Thus, many studies observed that this restriction treatment therapy produces cortical reorganization [65]. This showed a broad neural network involved in the occurrence of motor skills recovery in stroke patients; among these networks, we find the ipsilateral sensorimotor cortex, premotor area, supplementary motor area and parietal cortex [5,65]. Especially when CIMT was associated with TMS, we observed a significant increase in cortical reorganization through fMRI [52], and a significant increase in limb motor function recovery through MAL scale $[66,67]$. The cortical reorganization of the cerebral functions indicates potential changes that include mechanisms like those involved in the self-repair phenomenon $[68,69]$.

Liepert et al. [7] used TMS to study six patients before and after 14 days of CIMT, and observed neural recovery in the motor areas adjacent to the damaged neural region. In another study, Wittenberg et al. [59] restrained the unaffected upper limb of 17 post-stroke patients for 10 days and carried out the TMS three days prior to CIMT and 3 days after its completion. They noted that there were limitations in the use of TMS to measure physiological changes resulting from therapy, due to its inability to evoke responses in some patients with severe hemiparesis. These patients could recover motion through the action of some neurons in the corticospinal tract, but the TMS could not activate these neurons sufficiently.

Liepert et al. [70] restricted the unaffected upper limb movement in 13 hemiparetics post-Stroke patients. In this study they used the TMS and behavioral assessments one day before, one day, four days and six months after CIMT application. The researchers concluded that, one day after treatment, the injured hemisphere was $40 \%$ inactive while compared with the uninjured hemisphere. However, the injured hemisphere, after the first day of treatment, showed 37.5\% more neural activation than the uninjured hemisphere. This fact demonstrates that cortical activation in the injured hemisphere almost doubled between pre-CIMT and post-CIMT.

Before treatment, the excitability of the cortical area related to the muscles of the affected hand decreased. They suggested that this was probably due to the reduction in the use of this hand and to the presence of the lesion itself. After therapy, a nearly complete reversal of this abnormality was found; because of this, the researchers suggested that the most likely mechanism to justify this reversal is due to: reduced activity of local inhibitory interneurons, increased excitability in affected neurons and an extension of the neuronal tissue excitability in the affected hemisphere. However, regardless of the mechanisms, rehabilitation seems to lead to the growth of a large number of neurons in the affected premotor cortex.

When they analyzed the hemispheres 4 weeks after CIMT therapy, they observed that cortical activation in the affected hemisphere was greater than before CIMT therapy, and in the uninjured hemisphere a discrete increase occurred. Finally, when the researchers observed the patients six months after CIMT therapy, they found more cortical normalization in the affected hemisphere.

In another experiment, researchers observed 10 hemiparectic patients (i.e., 5 women and 5 men) with unilateral cortical and sub-cortical ischemic lesion. This treatment (i.e., CIMT) was used during 12 days and fMRI was used 48 hours before and 48 hours after treatment. The non-paretic upper limb was immobilized 10 hours per day with 30 minutes interval for lunch, dinner and personal hygiene. The study results demonstrated that, after CIMT, consistent activations in the primary sensorimotor cortex of the affected hemisphere occurred. Moreover, even extensive lesions appear not to inhibit the formation of neural circuits adjacent to the affected brain area. In spite of observing neural modulation effects predominantly in the affected hemisphere, one patient showed this modulation in the right and left hemispheres [71].

This result contradicts the study by Szaflarski et al. [17] in which they analyzed the cortical and behavioral changes in 4 patients (3 with right hemisphere damage and only 1 with left hemisphere damage) using functional magnetic resonance imaging (fMRI) and rating scales such as Action Research Arm Test (ARAT), upper-extremity portion of the Fugl-Meyer Assessment (FMA) and Motor Activity Log (MAL). The researchers said that in patients with unilateral brain lesions, the hemisphere opposite to the lesion is known to play an important role during the reorganization of manual functions.

Könönen et al. [72] analyzed 12 post-Stroke patients who were enrolled in a rehabilitation program during 2 
weeks. The patients remained with the upper limb immobilized for 10 hours a day. Moreover, the patients executed adapted exercise for 6 hours a day; the exercise gradually became more and more difficult throughout the two weeks. Researchers observed, through PET before and after CIMT therapy, that blood perfusion increased in the unaffected primary motor cortex and cingulated regions. Thus, in the affected hemisphere, the blood perfusions increased in the pre-motor area. In the pre-central gyrus, the lateral pre-motor area and medial supplementary motor area, they found an increase in perfusion not only in the affected hemisphere but also in the contralateral one. The researchers suggested that the increased electrical and metabolic activities found in both hemispheres occur before the execution of voluntary movements, especially of the more complex movements. According to them, the two hemispheres are activated, as they are intimately connected through the corpus callosum. However, a fact that deserved attention was that blood perfusion increased in two cerebellar hemispheres. The authors believe that, as the cerebellum plays a critical role in the coordination of voluntary movement and control of muscle tone and posture, perfusion increased so that these functions could be performed properly.

In addition to this, Tarkka et al. [25] agree with this information, as they also found plastic changes resulting from CIMT in four hemiparetic subjects using EEG as a method of evaluation. After treatment, EEG signals showed a large activation in the motor cortex, pre-motor cortex and the primary motor area in both hemispheres. The researchers suggest that changes in these locations may reflect an expansion or displacement of cortical regions, providing a recovery of the affected limb movement.

In addition to this, the neural functional state network is dependent on balance between inhibition and excitation received by the cortical areas [73]. Furthermore, the new functional architecture in the cortical reorganization is different among individuals with Stroke. This depends on lesion anatomy, biological age and chronicity [74]. Therefore, the mCIMT and CIMT have been effective therapies in upper limb movement rehabilitation and neural changes in post-Stroke patients [9].

\section{Conclusion}

We conclude that the behavioral and neuroimaging studies using mCIMT and CIMT promote cortical reorganization. Studies observed that many cortical areas like primary motor cortex, dorsal pre-motor cortex and supplementary motor area are activated by mCIMT and CIMT. However, there is no consensus about why some patients show a greater activation in the affected hemisphere, and why other patients experience a greater activation in the unaffected hemisphere. Consequently, the motor behavior in post-stroke patients is benefited from using mCIMT or CIMT; therefore, this therapy should be taken more into consideration by the professionals, due to its benefit. Finally, the world researchers still need uncountable studies to understand the gaps of cortical reorganization, and that restriction period and treatment are more effective with CIMT.

\section{REFERENCES}

[1] Y. Shi, H. J. Tian, K. H. Yang and Y. Zhao, "Modified Constraint-Induced Movement Therapy versus Traditional Rehabilitation in Patients with Upper-Extremity Dysfunction after Stroke: A Systematic Review and MetaAnalysis," Archives of Physical Medicine and Rehabilitation, Vol. 92, No. 6, 2011, pp. 972-982. http://dx.doi.org/10.1016/j.apmr.2010.12.036

[2] P. M. Rossini and G. D. Forno, "Neuronal Post-Stroke Plasticity in the Adult," Restorative Neurology and Neuroscience, Vol. 22, No. 3-5, 2004, pp. 193-206.

[3] S. J. Page and P. Levine, "Modified Constraint-Induced Therapy in Patients with Chronic Stroke Exhibiting Minimal Movement Ability in the Affected Arm,” Physical Therapy, Vol. 87, No. 7, 2007, pp. 872-878. http://dx.doi.org/10.2522/ptj.20060202

[4] A. Sterr, T. Elbert, I. Berthold, S. Kolbel, B. Rockstroh and E. Taub, "Longer versus Shorter Daily Constraint-Induced Movement Therapy of Chronic Hemiparesis: An Exploratory Study," Archives of Physical Medicine and Rehabilitation, Vol. 83, No. 10, 2002, pp. 1374-1377. http://dx.doi.org/10.1053/apmr.2002.35108

[5] E. Taub and G. Uswatte, "Contraint-Induced Movement Therapy: Bridging from the Primate Laboratory to the Stroke Rehabilitation Laboratory," Journal of Rehabilitation Medicine, Vol. 41, 2003, pp. 34-40. http://dx.doi.org/10.1080/16501960310010124

[6] W. H. R. Miltner H. Bauder, H. Sommer, M. Dettmers and E. Taub, "Effects of Constraint-Induced Movement Therapy on Patients with Chronic Motor Deficits after Stroke: A Replication,” Stroke, Vol. 30, No. 3, 1999, pp. 586-592. http://dx.doi.org/10.1161/01.STR.30.3.586

[7] J. Liepert, H. Bauder, H. R. Wolfgang, W. H. Miltner, E. Taub and C. Weiller, "Treatment-Induced Cortical Reorganization after Stroke in Humans,” Stroke, Vol. 31, No. 6, 2000, pp. 1210-1216. http://dx.doi.org/10.1161/01.STR.31.6.1210

[8] C. Y. Wu, L. L. Chuang, K. C. Lin, H. C. Chen and P. K. Tsay, "Randomized Trial of Distributed Constraint-Induced Therapy versus Bilateral Arm Training for the Rehabilitation of Upper-Limb Motor Control and Function after Stroke,” Neurorehabilitation and Neural Repair, Vol. 25, No. 2, 2011, pp. 130-139.

http://dx.doi.org/10.1177/1545968310380686

[9] S. W. Ward, "Plasticity and the Functional Reorganization of the Human Brain,” International Journal of Psychophysiology, Vol. 58, No. 2-3, 2005, pp. 158-161. http://dx.doi.org/10.1016/j.ijpsycho.2005.02.009

[10] C. E. Naylor and E. Bower, "Modified Constraint Induced Movement Therapy for Young Children with Hemiple- 
gic Cerebral Palsy: A Pilot Study,” Developmental Medicine \& Child Neurology, Vol. 47, No. 6, 2005, pp. 365369. http://dx.doi.org/10.1017/S0012162205000721

[11] F. Pulvermüller, B. Neininger, T. Elbert, B. Mohr, B. Rockstroh, P. Koebbel and E. Taub, "Constraint-Induced Therapy of Chronic Aphasia after Stroke,” Stroke, Vol. 32, No. 7, 2001, pp. 1621-1626. http://dx.doi.org/10.1161/01.STR.32.7.1621

[12] E. Taub, N. E. Miller, T. A. Novack, E. W. Cook, W. C. Fleming, C. S. Nepomuceno, J. S. Connell and J. E. Crago, "Technique to Improve Chronic Motor Deficit after Stroke," Archives of Physical Medicine and Rehabilitation, Vol. 74, No. 4, 1993, pp. 347-354.

[13] A. M. Gordon, J. S. Charles and S. L. Wolf, "Methods of Constraint-Induced Movement Therapy for Children with Hemiplegic Cerebral Palsy: Development of a ChildFriendly Intervention for Improving Upper-Extremity Function," Archives of Physical Medicine and Rehabilitation, Vol. 74, No. 4, 2005, pp. 837-844. http://dx.doi.org/10.1016/j.apmr.2004.10.008

[14] D. M. Morris, E. Taub and V. W. Mark, "Contraint-Induced Movement Therapy. Characterizing the Intervention Protocol," Europa Medicophysica, Vol. 42, No. 3, 2006, pp. 257-268.

[15] E. Taub, S. L. Ramey, S. DeLuca and K. Echols, "Efficacy of Constraint-Induced Movement Therapy for Children with Cerebral Palsy with Asymmetric Motor Impairment,” Pediatrics, Vol. 113, No. 2, 2004, pp. 305-312. http://dx.doi.org/10.1542/peds.113.2.305

[16] S. J. Page, S. Sisto, P. Levine, M. V. Johnston and M. Hughes, "Modified Contraint Induced Therapy: A Randomized Feasibility and Efficacy Study," Journal of Rehabilitation Research \& Development, Vol. 38, No. 5, 2001, pp. 583-590.

[17] J. P. Szaflarski, S. J. Page, B. M. Kissela, J. H. Lee, P. Levine and S. M. Strakowski, "Cortical Reorganization Following Modified Constraint-Induced Movement Therapy: A Study of 4 Patients with Chronic Stroke,” Archives of Physical Medicine and Rehabilitation, Vol. 87, No. 8, 2006, pp. 1052-1058. http://dx.doi.org/10.1016/j.apmr.2006.04.018

[18] S. J. Page, S. Sisto, P. Levine and R. E. McGrath, "Efficacy of Modified Constraint-Induced Movement Therapy in Chronic Stroke: A Single-Blinded Randomized Controlled Trial," Archives of Physical Medicine and Rehabilitation, Vol. 85, No. 1, 2004, pp. 14-18.

[19] M. S. George, Z. Nahas, S. H. Lisanby, T. Schlaepfer, F. A. Kozel and B. D. Greenberg, "Transcranial Magnetic Stimulation," Neurosurgery Clinics of North America, Vol. 14, No. 2, 2003, pp. 283-301. http://dx.doi.org/10.1016/S1042-3680(02)00120-1

[20] K. Wendel, O. Väisänen, J. Malmivuo, N. G. Gencer, B. Vanrumste, P. Durka, R. Magjarevic, S. Supek, L. M. Pascu, H. Fontenelle and R. G. P. Menendez, "EEG/MEG Source Imaging: Methods, Challenges, and Open Issues,” Computational Intelligence and Neuroscience, Vol. 2009, No. 12, 2009, pp. 1-12. http://dx.doi.org/10.1155/2009/656092

[21] V. R. Badrakalimuthu, R. Swamiraju and H. Waal, "EEG in Psychiatric Practice: To Do or Not To Do?” Advances in Psychiatric Treatment, Vol. 17, No. 2, 2011, pp. 114121. http://dx.doi.org/10.1192/apt.bp.109.006916

[22] B. Draganski, C. Gases, V. Busch, G. Schuierer, U. Bogdahn and A. May, "Neuroplasticity: Changes in Grey Matter Induced by Training,” Nature, Vol. 427, 2004, pp. 311-312. http://dx.doi.org/10.1038/427311a

[23] T. M. Lau, J. T. Gwin, K. G. McDowell and D. P. Ferris, "Weighted Phase Lag Index Stability as an Artifact Resistant Measure to Detect Cognitive EEG Activity during Locomotion,” Journal of NeuroEngineering and Rehabilitation, Vol. 9, No. 42, 2012, p. 47.

[24] J. R. Hughes and E. R. John, "Conventional and Quantitative Electroencephalography and Psichiatry,” The Journal of Neuropsychiatry and Clinical Neurosciences, Vol. 11, No. 2, 1999, pp. 190-208.

[25] I. M. Tarkka, M. Kononen, K. Pitka, J. Sivenius and E. Mervaala, "Alterations in Cortical Excitability in Chronic Stroke after Constraint-Induced Movement Therapy,” Neurological Research, Vol. 30, No. 5, 2008, pp. 504-510. http://dx.doi.org/10.1179/016164107X252519

[26] A. T. Barker, R. Jalinous and I. L. Freeston, "Noinvasive Magnetic Stimulation of Human Motor Cortex," Lancet, Vol. 1, No. 8437, 1985, pp. 1106-1107. http://dx.doi.org/10.1016/S0140-6736(85)92413-4

[27] M. Hallett. "Transcranial Magnetic Stimulation and the Human Brain,” Nature, Vol. 406, No. 6792, 2000, pp. 147-150.

[28] S. Machado, B. Velasques, M. Cunha, L. Basile, H. Budde, M. Cagy, R. Piedade and P. Ribeiro. “Aplicações Terapêuticas da Estimulação Cerebral por Corrente Contínua na Neuroreabilitação Clínica,” Revista Neurociências, Vol. 17, No. 3, 2009, pp. 298-300.

[29] F. Fregni, S. C. Schachter and A. Pacual-Leone, "Transcranial Magnetic Stimulation Treatment for Epipepsy: Can It Also Improve Depression and Vice Versa?” Epilepsy \& Behavior, Vol. 7, No. 2, 2005, pp. 182-189. http://dx.doi.org/10.1016/j.yebeh.2005.06.004

[30] S. Machado, F. Paes, B. Velasques, S. Teixeira, R. Piedade, P. Ribeiro, A. E. Nardi and O. Arias-Carrión, "Is rTMS an Effective Therapeutic Strategy That Can Be Used to Treat Anxiety Disorders?” Neuropharmacology, Vol. 62, No. 1, 2011, pp. 125-134.

http://dx.doi.org/10.1016/j.neuropharm.2011.07.024

[31] A. Pascual-Leone, V. Walsh and J. Rothwell, "Transcranial Magnetic Stimulation in Cognitive NeuroscienceVirtual Lesion, Chronometry, and Functional Connectivity," Current Opinion in Neurobiology, Vol. 10, No. 2, 2000, pp. 232-237. http://dx.doi.org/10.1016/S0959-4388(00)00081-7

[32] Z. Nahas, X. Li, J. H. Chae, N. C. Oliver, B. Anderson, B. Kapp and M. S. George, "What Does ECS Stand for? Repetitive Transcranial Magnetic Stimulation in Depression,” Epilepsy \& Behavior, Vol. 2, No. 3, 2001, 2001, p. 375.

[33] A. Valentin, R. Arunachalam, A. Mesquita-Rodrigues, J. J. Garcia, M. P. Richardson, K. R. Mills and G. Alarcon, "Late EEG Responses Triggered by Transcranial Magnetic Stimulation (TMS) in the Evaluation of Focal Epi- 
lepsy,” Epilepsia, Vol. 49, No. 3, 2007, pp. 470-480. http://dx.doi.org/10.1111/j.1528-1167.2007.01418.x

[34] N. Osaka, Y. Otsuka, N. Hirose, T. Ikeda, T. Mima, H. Fukuyama and M. Osaka, "Transcranial Magnetic Stimulation (TMS) Applied to Left Dorsolateral Prefrontal Cortex Disrupts Verbal Working Memory Performance in Humans,” Neuroscience Letters, Vol. 418, No. 3, 2007, pp. 232-235. http://dx.doi.org/10.1016/j.neulet.2007.01.087

[35] S. Pallanti and S. Bernardi. "Neurobiology of Repeated Transcranial Magnetic Stimulation in the Treatment of Anxiety: A Critical Review,” International Clinical Psychopharmacology, Vol. 24, No. 4, 2009, pp. 163-173. http://dx.doi.org/10.1097/YIC.0b013e32832c2639

[36] F. Paes, S. Machado, O. Arias-Carrión, B. Velasques, S. Teixeira, H. Budde, M. Cagy, R. Piedade, P. Ribeiro, J. P. Huston, A. T. Sack and A. E. Nardi, "The Value of Repetitive Transcranial Magnetic Stimulation (rTMS) for the Treatment of Anxiety Disorders: An Integrative Review," CNS \& Neurological Disorders-Drug Targets, Vol. 10, No. 5, 2011, pp. 610-620. http://dx.doi.org/10.2174/187152711796234943

[37] G.M. Pohost and A.V. Ratner, "Nuclear Magnetic Resonance. Potential Applications in Clinical Cardiology," JAMA, Vol. 251, No. 10, 1984, pp. 1304-1309. http://dx.doi.org/10.1001/jama.1984.03340340044026

[38] N. R. Logothetis, J. Pauls, M. Augath, T. Trinath and A. Oeltermann, "Neurophysiological Investigation of the Basis of the fMRI Signal,” Nature, Vol. 412, No. 6843, 2011, pp. 150-157. http://dx.doi.org/10.1038/35084005

[39] M. S. Sercheli, E. Bilevicius, A. Alessio, H. Ozelo, F. R. Pereira, J. M. Rondina, F. R. Cendes and J. Covolan, "EEG Spike Source Localization before and after Surgery for Temporal Lobe Epilepsy: A BOLD EEG-fMRI and Independent Component Analysis Study,” Brazilian Journal of Medical and Biological Research, Vol. 42. No. 6, 2009, pp. 582-587. http://dx.doi.org/10.1590/S0100-879X2009000600017

[40] M. S. Meneses, J. C. M. Pedroso, R. F. Fuzza and J. B. Milano, "Comparative Analysis of Human Brains Slices with Three Different Staining Techniques," Arquivos de Neuro-Psiquiatria, Vol. 62. No. 2, 2004, pp. 276-281. http://dx.doi.org/10.1590/S0004-282X2004000200016

[41] S. P. Ku, A. S. Tolias, N. K. Logothetis and J. Goense, "fMRI of the Face-Processing Network in the Ventral Temporal Lobe of Awake and Anesthetized Macaques," Neuron, Vol. 70. No. 2, 2011, pp. 352-362. http://dx.doi.org/10.1016/j.neuron.2011.02.048

[42] Y. H. Kim, J. W. Park, M. H. Ko and P. K. Lee, "Plastic Changes of Motor Network after Constraint-Induced Movement Therapy," Yonsei Medical Journal, Vol. 45. No. 2, 2004, pp. 241-246.

[43] J. C. Eliassen, E. L. Boespflug, M. Lamy, J. Allendorfer, W. J. Chu and J. P. Szaflarski, "Brain-Mapping Techniques for Evaluating Poststroke Recovery and Rehabilitation: A Review," Topics in Stroke Rehabilitation, Vol. 15. No. 5, 2008, pp. 427-450. http://dx.doi.org/10.1310/tsr1505-427

[44] M. A. Macleod, G. H. Adkisson, M. J. Fox and R. P.
Pearson, “99Tcm-HMPAO Single Photon Emission Tomography in the Diagnosis of Cerebral Barotraumas," British Journal of Radiology, Vol. 61. No. 732, 1988, pp. 1106-1109.

http://dx.doi.org/10.1259/0007-1285-61-732-1106

[45] G. Muehllehner and J. S. Karp, "Positron Emission Tomography," Physics in Medicine and Biology, Vol. 51, No. 13, 2006, pp. 117-137. http://dx.doi.org/10.1088/0031-9155/51/13/R08

[46] F. Lomeña and M. Soler, "Clinical Application of Pet," Brazilian Archives of Biology and Technology, Vol. 48, No. 2, 2005, pp. 179-183. http://dx.doi.org/10.1590/S1516-89132005000700027

[47] H. Lundqvist, M. Lubberink and V. Tolmachev, "Positron Emission Tomography,” European Journal of Physics, Vol. 19, No. 6, 1998, pp. 537-552. http://dx.doi.org/10.1088/0143-0807/19/6/007

[48] O. Arias-Carrion, S. Machado, F. Paes, B. Velasques, S. Teixeira, L. Cardenas-Morales, R. Piedade, R. Ribeiro and A. E. Nardi, "Is rTMS an effective Therapeutic Strategy That Can Be Used to Treat Parkinson's Disease?” CNS \& Neurological Disorders-Drug Targets, Vol. 10. No. 6, 2011, pp. 693-702.

[49] S. Casarotto, L. J. R. Lauro, V. Bellina, A. G. Casali, M. Rosanova, A. Pigorini, S. Defendi, M. Mariotti and M. Massimini, "EEG Responses to TMS Are Sensitive to Changes in the Perturbation Parameters and Repeatable over Time,” PLoS ONE, Vol. 5. No. 4, 2010, Article ID: 10281. http://dx.doi.org/10.1371/journal.pone.0010281

[50] E. Taub, G. Uswatte, D. K. King, D. Morris, J. E. Crago and A. Chatterjee, "A Placebo-Controlled Trial of Constraint-Induced Movement Therapy for Upper Extremity After Stroke,” Stroke, Vol. 37, 2006, pp. 1045-1049. http://dx.doi.org/10.1161/01.STR.0000206463.66461.97

[51] A. Kunkel, B. Kopp, G.K. Miiller, A. Villringer, E. Taub and H. Flor, "Constraint-Induced Movement Therapy for Motor Recovery in Chronic Stroke Patients," Archives of Physical Medicine and Rehabilitation, Vol. 80, No. 6, 1999, pp. 624-626. http://dx.doi.org/10.1016/S0003-9993(99)90163-6

[52] E. Taub, G. Uswatt and R. Pidikiti, “Constraint-Inducel Movement Therapy: A New Family of Techniques with Broad Application to Physical Rehabilitation-A Clinical Review," Journal of Rehabilitation Research \& Development, Vol. 36. No. 3, 1999, pp. 237-251.

[53] S. J. Page and P. L. Levine. "Modified Constraint Induced Therapy Extension: Using Remote Technologies to Improve Function," Archives of Physical Medicine and Rehabilitation, Vol. 36, No. 3, 2007, pp. 922-927. http://dx.doi.org/10.1016/j.apmr.2007.03.038

[54] S. J. Page, S. Sisto, M. V. Johnston and P. Levine, "Modified Constraint-Induced Therapy after Subacute Stroke: A Preliminary Study," Neurorehabilitation and Neural Repair, Vol. 16, No. 3, 2002, pp. 290-295.

[55] M. Hosomi, T. Koyama, T. Takebayashi, S. Terayama, N. Kodama, K. Matsumoto and K. Domen, “A Modified Method for Constraint-Induced Movement Therapy: A Supervised Self-Training Protocol," Journal of Stroke and Cerebrovascular Diseases, Vol. 21. No. 8, 2011, pp. 
767-75.

[56] C. Y. Wu, Y. W. Hsieh, K. C. Lin, L. L. Chuang, Y. F. Chang, H. L. Liu, C. Chen, K. H. Lin and Y. Y. Wai, "Brain Reorganization after Bilateral Arm Training and Distributed Constraint-Induced Therapy in Stroke Patients: A Preliminary Functional Magnetic Resonance Imaging Study,” Chang Gung Medical Journal, Vol. 33, No. 6, 2010, pp. 628-638.

[57] S. L. Wolf, P. A, Thompson, C. Winstein, J. P. Miller, S. R. Blanton, D. S. N. Larsen, D. M. Morris, G. Usmatte. E. Taub, K. E. Light and L. Sawaki, "The EXCITE Stroke Trial Comparing Early and Delayed Constraint-Induced Movement Therapy,” Stroke, Vol. 41, No. 10, 2010, pp. 2309-2315. http://dx.doi.org/10.1161/STROKEAHA.110.588723

[58] J. Biernaskie, G. Chernenko and D. Corbett, "Efficacy of Rehabilitative Experience Declines with Time after Focal Ischemic Brain Injury,” The Journal of Neuroscience, Vol. 24, No. 5, 2004, pp. 1245-1254. http://dx.doi.org/10.1523/JNEUROSCI.3834-03.2004

[59] G. F. Wittenberg, R. Chen, K. Ishii, K. O. Bushara, E. Taub, L. H. Gerber, M. Hallett and L. G. Cohen, "Constraint-Induced Therapy in Stroke: Magnetic-Stimulation Motor Maps and Cerebral Activation,” Neurorehabilitation and Neural Repair, Vol. 17, No. 1, 2003, pp. 48-57. http://dx.doi.org/10.1177/0888439002250456

[60] C. J. Winstein, C. J. Miller, J. P. Blanton, E. Taub, G. Uswatte, D. Morris, D. Nichols and S. Wolf, "Methods for a Multisite Randomized Trial to Investigate the Effect of Constraint-Induced Movement Therapy in Improving Upper Extremity Function among Adults Recovering from a Cerebrovascular Stroke,” Neurorehabilitation and Neural Repair, Vol. 17, No. 3, 2003, pp. 137-152. http://dx.doi.org/10.1177/0888439003255511

[61] J. D. Schaechter, E. Kraft, T. S. Hilliard, R. M. Dijkhuizen, T. Benner, S. P. Finklestein, B. R, Rosen and S. C. Cramer, "Motor Recovery and Cortical Reorganization after Constraint-Induced Movement Therapy in Stroke Patients: A Preliminary Study,” Neurorehabilitation and Neural Repair, Vol. 16, No. 4, 2002, pp. 326-328. http://dx.doi.org/10.1177/154596830201600403

[62] S. Blanton, S. L. Wolf. “An Application of Upper-Extremity Constraint-Induced Movement Therapy in a Patient with Subacute Stroke,” Physical Therapy, Vol. 79, No. 9, 1999, pp. 847-853.

[63] S. Hakkennes and J. L. Keating, "Constraint-Induced Movement Therapy Following Stroke: A Systematic Review of Randomised Controlled Trials,” Australian Journal of Physiotherapy, Vol. 51, No. 4, 2005, pp. 221-231. http://dx.doi.org/10.1016/S0004-9514(05)70003-9

[64] W. C. Huang, Y. J. Chen, C. L. Chien, H. Kashima and K. C. Lin, "Constraint-Induced Movement Therapy as a Paradigm of Translational Research in Neurorehabilitation: Reviews and Prospects," American Journal of Translational Research, Vol. 3. No. 1, 2011, pp. 48-60.

[65] A. Feydy, R. Carlier, A. Roby-Brami, B. Bussel, F, Cazalis, L. Pierot, Y. Burnod and M. A. Maier, "Longitudinal Study of Motor Recovery after Stroke: Recruitment and Focusing of Brain Activation,” Stroke, Vol. 33 No. 6,
2002, pp. 1610-1617.

http://dx.doi.org/10.1161/01.STR.0000017100.68294.52

[66] B. Sheng and M. Lin, “A Longitudinal Study of Functional Magnetic Resonance Imaging in Upper-Limb Hemiplegia after Stroke Treated with Constraint-Induced Movement Therapy," Brain Injury, Vol. 23. No. 1, 2009, pp. 65-70. http://dx.doi.org/10.1080/02699050802635299

[67] C. E. Levy, D. S. Nichols, P. M. Schmalbrock, P. Keller and D. W. Chakeres, "Functional MRI Evidence of Cortical Reorganization in Upper-Limb Stroke Hemiplegia Treated with Constraint-Induced Movement Therapy,” American Journal of Physical Medicine \& Rehabilitation, Vol. 80, No. 1, 2001, pp. 4-12.

http://dx.doi.org/10.1097/00002060-200101000-00003

[68] P. Rossini, P. Mand and S. Rossi. "Transcranial Magnetic Stimulation: Diagnostic, Therapeutic, and Research Potential,” Neurology, Vol. 68. No. 7, 2007, pp. 484-488. http://dx.doi.org/10.1212/01.wnl.0000250268.13789.b2

[69] Z. R. Hunter, "Plasticity of the Adult Human Brain and Motor Recovery after Stroke,” Institute of Cognitive Science, Vol. 36, No. 213, 2005, p. 5:1.

[70] J. Liepert, W. H. R. Miltner, H. Bauder, M. Sommer, C. Dettmers, E. Taub and C. Weiller, "Motor Cortex Plasticity during Constraint-Induced Movement Therapy in Stroke Patients," Neuroscience Letters, Vol. 250, No. 1, 1998, pp. 5-8. http://dx.doi.org/10.1016/S0304-3940(98)00386-3

[71] H. Juenger, M. Linder-Lucht, M. Walther, S. Berweck, V. M. Mall and S. Taudt, "Cortical Neuromodulation by Constraint-Induced Movement Therapy in Congenital Hemiparesis: An fMRI Study,” Neuropediatrics, Vol. 38, No. 3, 2007, pp. 130-136. http://dx.doi.org/10.1055/s-2007-985904

[72] M. Könönen, J. T. Kuikka, M. Husso-Saastamoinen, E. Vanninen, R. Vanninen, S. Soimakallio, E. Mervaala, J. Sivenius, K. Pitkänen and I. M. Tarkka, "Increased Perfusion in Motor Areas after Constraint-Induced Movement Therapy in Chronic Stroke: A Single-Photon Emission Computerized Tomography Study,” Journal of Cerebral Blood Flow \& Metabolism, Vol. 25, No. 12, 2005, pp. 1668-1674. http://dx.doi.org/10.1038/sj.jcbfm.9600158

[73] M. N. Economo and J. A. White, "Membrane Properties and the Balance between Excitation and Inhibition Control Gamma-Frequency Oscillations Arising from Feedback Inhibition,” PLOS Computational Biology, Vol. 8, No. 1, 2012, Article ID: 1002354. http://dx.doi.org/10.1371/journal.pcbi.1002354

[74] A. Kleim and T. A. Jones, "Principles of Experience-Dependent Neural Plasticity: Implications for Rehabilitation after Brain Damage,” Journal of Speech, Language, and Hearing Research, Vol. 51, No. 1, 2008, pp. 225-239. http://dx.doi.org/10.1044/1092-4388(2008/018)

[75] C. Boake, E.A. Noser, T. Ro, S. Baraniuk, M. Gaber, R. Johnson, E. T. Salmeron, T. M. Tran, J. M. Lai, E. Taub, L. A. Moye, J. C. Grotta and H. S. Levin, "ConstraintInduced Movement Therapy during Early Stroke Rehabilitation," Neurorehabilitation and Neural Repair, Vol. 21, No. 1, 2007, pp. 14-24.

http://dx.doi.org/10.1177/1545968306291858 